\section{Exakte Ergebnisse durch reines Reagens}

D ie mikrobielle Kontamination von Forschungsreagenzien ist ein weit verbreitetes Problem. Vor allem die daraus resultierenden erhöhten Endotoxinwerte können in der immunologischen Forschung zu Fehlinterpretationen bei Zellkulturassays und Tierversuchen führen. Um solche Artefakte ausschließen zu können, arbeitet das Regensburger Biotech-Unternehmen Profos an der Entwicklung qualitativ hochwertiger, Endotoxin-freier Produkte. Erstes Ergebnis dieser Forschung ist das neue
EndoGrade ${ }^{\mathrm{TM}}$ Ovalbumin, das seit August 2007 auf dem Markt ist.

Das Glykoprotein Ovalbumin ist eines der meistgenutzten Proteine in Tierversuchen und zudem ein etabliertes Modellallergen in der Asthmaforschung. Kommerziell erhältliches Ovalbumin ist jedoch oft mit Lipopolysacchariden kontaminiert, die aufgrund ihrer immunmodulierenden Eigenschaften die Versuchsergebnisse verzerren können. Profos ist es jetzt gelungen, mit der patentierten EndoTrap $^{\circledR}$-Technologie Ovalbumin her- zustellen, dessen Endotoxingehalt unter 1 EU/mg liegt. Somit können Nebeneffekte durch Lipopolysaccharide ausgeschlossen werden.

Dem neuen Ovalbumin sollen in naher Zukunft weitere Endotoxin-freie EndoGrade ${ }^{\mathrm{TM}}$-Reagenzien folgen. Für weitere Informationen zu den Produkten von Profos kontaktieren Sie bitte Dr. Stephanie Steck, Tel. (09 41) 94262 0, E-Mail: stephanie.steck@profos.de, oder besuchen unter www.profos.de die Homepage des Unternehmens.

Nach Informationen von Profos, Regensburg

\title{
Argumente für die Hochdosistherapie
}

$M$ etaanalysen wie auch eigene Auswertungen zeigen nach den Worten von Prof. Dr. Hans-Jørgen Malling, Kopenhagen, Dänemark, nach wie vor eine therapeutische Überlegenheit der subkutanen (SCIT) gegenüber der sublingualen Immuntherapie (SLIT). Allerdings sei die SLIT besser verträglich, gestand Malling zu. „Entscheidend bei beiden Therapieformen ist aber die Höhe der Allergenmenge pro Einzeldosis“, erklärte er und belegte diese Behauptung mit Dosis-Wirkungs-Studien mit standardisierten Allergenextrakten.

Dass die Höhe der Einzeldosis der wichtigste Parameter für die Wirksamkeit ist, sei auch ein Fazit des FASITTreffens Ende 2006 gewesen, informierte Prof. Dr. Ulrich Wahn, Berlin. FASIT steht für „The future of the allergist and specific immunotherapy " und wird von der EAACI und dem Unternehmen Allergopharma unterstützt. Renommierte Experten diskutieren im Rahmen dieser Initiative State of the Art und Zukunft der spezifischen Hyposensibilisierung. Diese dürfte in den kommenden Jahren vor allem durch die rekombinanten Allergene und die Weiterentwicklung hypoallergener Therapieoptionen geprägt sein, fasste Wahn die Mehrheitsmeinung seiner Kollegen zusammen.

Die klinische Wirksamkeit und gute Verträglichkeit rekombinanter Allergene wurde bereits in kontrollierten klinischen
Studien gezeigt, erläuterte Priv.-Doz. Dr. Peter Eng, Aarau, Schweiz. Sogar hypoallergene rekombinante Allergene wurden schon mit Erfolg klinisch erprobt. Sie könnten mit einer verminderten Injektionszahl und in hoher Einzeldosierung gegeben werden, erklärte Eng, dabei seien sie ähnlich verträglich und sogar besser wirksam als konventionelle Präparate.

Das Hochdosiskonzept funktioniert auch bei der SLIT. Priv.-Doz. Dr. Jörg Kleine-Tebbe, Berlin, stellte als Beleg die Ergebnisse einer über eineinhalb Jahre durchgeführten Doppelblindstudie vor, in der Patienten mit einer Allergie gegen Gräserpollen randomisiert mit dem Hochdosis-SLIT-Präparat AllerSlit $^{\circledR}$ forte oder Plazebo behandelt worden waren. Das Studienpräparat enthält eine Allergenmischung aus sechs verschiedenen Gräsern und ist ELISA-standardisiert auf $40 \mu \mathrm{g}$ Gruppe-5-Allergen pro Einzeldosis. Am Ende der Studie ergab sich eine statisch signifikante und klinisch relevante Überlegenheit des neuen SLIT-Präparats, berichtete Kleine-Tebbe.

$b k$

Satellitensymposium „High-dose-SIT

- first choice against allergy“. XXVI Congress of the European Academy of Allergology and Clinical Immunology (EAACI), Göteborg, Schweden, 11.-13. Juni 2007. Veranstalter: Allergopharma, Reinbek

\section{Service Box sorgt für mehr} Compliance

Das Unternehmen Allergopharma hat sein Serviceangebot zur sublingualen Immuntherapie (SLIT) erweitert: Die neue „AllerSlit ${ }^{\circledR}$ forte Service Box" soll die Compliance der Patienten fördern und so den langfristigen Erfolg der Behandlung sichern. Die Box enthält eine ausführliche Informationsbroschüre zur hochdosierten SLIT, ein Therapietagebuch zur Dokumentation sowie ein praktisches Etui zur Aufbewahrung der SingleDose Strips. Clou ist eine kleine Sanduhr, die hilft, die AllerSlit ${ }^{\circledR}$-Dosis die empfohlenen drei Minuten unter der Zunge zu halten.

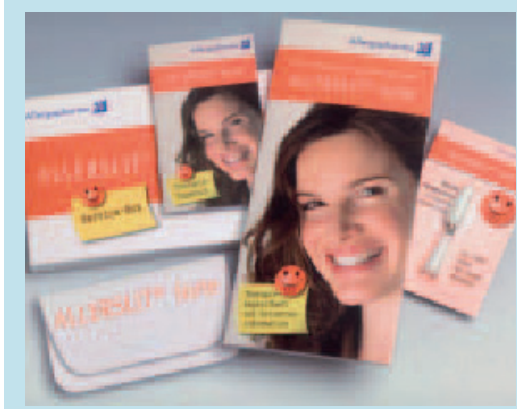

Allergologen können die Service Box über den Allergopharma-Außendienst kostenlos für ihre Patienten anfordern. Weitere Informationen zur hoch dosierten SLIT sind über die passwortgeschützte Website www.allerslit.de abrufbar.

Nach Informationen von Allergopharma, Reinbek 